\title{
Time-division multiple access based intra-body communication for wearable health tracker
}

\begin{abstract}
In this project, a medium access control utilizing the technique of time-division multiple access was developed for the intra-body communication. A prototype of a wearable health tracker was designed based on a commercially available development kit. The prototype consists of a base unit and three sensor nodes to form a single-hop star topology body area network. Each of the sensor nodes can be interfaced with analog or digital type of wearable sensors to measure human physiological data.
\end{abstract}

Keyword: BAN; Intra-body communication (IBC); MAC; TDMA 